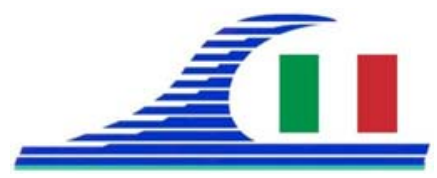

Conférence Méditerranéenne Côtière et Maritime EDITION 3, FERRARA, ITALIA (2015)

Coastal and Maritime Mediterranean Conference

Disponible en ligne - http://www.paralia.fr - Available online

\title{
Waves and currents propagation through porous media: model experiments and scale effects
}

\author{
Gwendoline ARNAUD ${ }^{1,2}$, Vincent REY ${ }^{1}$, Damien SOUS ${ }^{1}$, \\ Julien TOUBOUL ${ }^{1}$, Fabrice GOUAUD ${ }^{2}$
}

1. Université de Toulon, CNRS/INSU, IRD, Mediterranean Institute of Oceanography (MIO), UM 110, 83957 La Garde, France.

Aix Marseille Université, CNRS/INSU, IRD, Mediterranean Institute of Oceanography (MIO), UM 110, 13288 Marseille, France.

garnaud@univ-tln.fr ; rey@univ-tln.fr ; sous@univ-tln.fr ; touboul@univ-tln.fr

2. ACRI-In, 260, route du Pin Montard Sophia-Antipolis F-06410 Biot, France.

Fabrice.Gouaud@acri-in.fr

\begin{abstract}
:
This note is related to the scaling effects of porous media consisting in emerging vertical cylinder array. Wave and current experiments were carried out in a 10m-long flume. Three cylinder diameters are considered in order to study the role of the specific surface while keeping the porosity constant. Both porous media length and water depth are selected to scale with the cylinder diameters. The collected data show the influence of the specific surface on interference process via the reflection coefficient. Then, a theory of wave amplitude attenuation is developed using a force expression based on the work of O'BRIEN \& MORISON, 1952. This model is calibrated with drag and inertia coefficients. Finally, scaling emphasizes the importance of the flow regime within the porous media to verify flow similarity.
\end{abstract}

Keywords: Porous structure, specific surface, wave, current, scale effects, reflection, dissipation.

\section{Introduction}

Physical model in basin or wave flume are generally used to mimic fluid flow dynamics using scaling laws. In this context, several studies exist concerning the scale effects through various porous structures (DELMONTE, 1972; PEREZ-ROMERO et al., 2009; ANDERSEN et al., 2011; ALTOMARE \& GIRONELLA, 2014; and references herein). However, the influence of specific surface is rarely explicitly highlighted for scaling analysis although this parameter changes with the scale even if the porosity is constant. This work presents a study of scaling effect comparing three model porous media keeping the porosity constant while the specific surface, defined as a fluid-solid surface contact per volume unit (GUYON et al., 1991), varies with the cylinder diameter. 
Côtes méditerranéennes menacées :

Risques et défis dans le contexte du changement climatique

\section{Experimental set-up}

The laboratory flume (SeaTech, University of Toulon, France) used for the experiments is $10 \mathrm{~m}$ long, $0.30 \mathrm{~m}$ wide and $0.50 \mathrm{~m}$ high. The model porous media are made of a regular array of emerging vertical cylinders of constant diameter. The cylinders are regularly disposed along two perpendicular axes forming a $45^{\circ}$ angle with the longitudinal axis. The entire experimental set-up, including the positioning of sensors, are detailed in the previous study dealing with regular waves propagation through porous media (ARNAUD et al., 2014). Regular waves of frequency in the range 0.2-1.8 $\mathrm{Hz}$ are considered.

Table 1. Summary of experiment conditions.

\begin{tabular}{lllll}
\hline $\begin{array}{l}\text { Cylinder } \\
\text { diameter } \mathbf{D}(\mathbf{m})\end{array}$ & $\begin{array}{l}\text { Medium porous } \\
\text { length } \mathbf{L}(\mathbf{m})\end{array}$ & $\begin{array}{l}\text { Water } \\
\text { depth } \boldsymbol{h}(\boldsymbol{m})\end{array}$ & $\begin{array}{l}\text { Specific surface } \\
\boldsymbol{s}\left(\mathbf{m}^{-1}\right)\end{array}$ & Porosity $\boldsymbol{~}$ \\
\hline 0.050 & 1.80 & 0.36 & 22 & 0.7 \\
0.032 & 1.15 & 0.23 & 33 & 0.7 \\
0.020 & 0.72 & 0.14 & 52 & 0.7 \\
\hline
\end{tabular}

\section{Scale effects on the wave reflection and transmission}

\subsection{The linear model}

The linear potential theory is used to describe the wave propagation in porous media. Details are presented in ARNAUD et al. (2014). The velocity potential for each domain is expressed by the following equation:

$$
\Phi_{j}(x, z, t)=\varphi_{j}(x, z) \sin (\omega t)=\frac{a_{j}^{ \pm} \omega}{k_{j}} \frac{\cosh \left[k_{j}(z+h)\right]}{\sinh \left(k_{j} h\right)} \sin \left(\omega t \pm k_{j} x\right)
$$

where a is the wave amplitude, $k_{j}$ is the wave number and $\omega=2 \pi \mathrm{f}=2 \pi / \mathrm{T}$ is the angular frequency. Moreover, the influence of the evanescent modes, which appear in the presence of vertical discontinuities, was found to be negligible in the present work. The wave amplitude coefficients $\mathrm{a}_{\mathrm{j}}^{ \pm}$vary within the porous medium with the longitudinal propagation due to the energy dissipation process detailed in the following sub-section (3.2). Damping is neglected on both parts of the porous medium thus the coefficients $\mathrm{a}_{\mathrm{j}}{ }^{ \pm}$ are constant in these two domains. The present problem is solved using an integral matching method of the pressure and momentum continuity conditions at the porous vertical interfaces $\mathrm{x}=0$ and $\mathrm{x}=\mathrm{L}$ (YU \& CHWANG, 1994):

$\varphi_{i}=S_{r} \varphi_{j} \quad \& \quad \frac{\partial \varphi_{i}}{\partial x}=\gamma \frac{\partial \varphi_{j}}{\partial x}$

where index $i$ corresponds to either upstream $(x \leq 0)$ or downstream $(x \geq L)$ domains on each side of the porous medium and index $j$ to the porous medium. The reactance $S_{r}$ (eq. 5) involves the added mass coefficient $C_{m}$ adjusted to fit the theoretical model with 
the interference process of experimental data. The general form of the dispersion relation keeping only the inertial effects (YU \& CHWANG, 1994) is written:

$\omega^{2} S_{r}=g k_{j} \tanh \left(k_{j} h\right)$, with $: S_{r}=1+C_{m} \frac{1-\gamma}{\gamma}$

where $\gamma$ is the media porosity and g is the gravity acceleration. $S_{r}=1$ for upstream and downstream domains. The relation dispersion defined in eq. (3) implies that the wavelength in the porous medium depends on the added mass $\mathrm{C}_{\mathrm{m}}$.

\subsection{Wave amplitude evolution}

The energy dissipation rate is assumed to be the sum of the drag effect of each of the cylinders. This rate is calculated along an elementary length $\Delta \mathrm{l}$ with the hypothesis $\mathrm{L}>>\Delta \mathrm{l}>>\mathrm{D}$. The drag stress due to one cylinder is given by (eq. 4).

$$
F_{d}=\int_{-h}^{0} \frac{1}{2} \rho C_{d, s} D U|U| d z=\frac{1}{8} \rho C_{d, s} D a^{2} \omega^{2} \frac{2 k_{j} h+\cosh \left(4 k_{j} h\right)}{k \sinh ^{2}\left(k_{j} h\right)}
$$

where $\rho$ is the fluid density, $C_{d, s}$ is the drag coefficient for a single cylinder, $D$ is the cylinder diameter, $U$ is the fluid velocity, $h$ is the water depth and $a$ is the wave amplitude $\mathrm{a}_{\mathrm{j}}$. The dissipated energy $\Delta \mathrm{E}_{\mathrm{N}}$ (eq. 5) related to drag force is defined as the integrand over a wave period $\mathrm{T}_{\mathrm{w}}$ of $\mathrm{F}_{\mathrm{d}}$.

The cylinders number is calculated using the porosity parameter defined by: $N=(1-\gamma) \frac{4 \Delta l B}{\pi D^{2}}$.

$\Delta E_{N}=N \int_{0}^{T_{w}} F_{d} U d t=-\frac{16}{9 \pi}(1-\gamma) \rho C_{d, s} \frac{a_{j}^{3} \Delta l B}{D} g \tanh \left(k_{j} h\right)\left(\frac{3}{\sinh ^{2}\left(k_{j} h\right)}+1\right)$

where $\mathrm{B}$ is the porous width.

Moreover, another expression of dissipated energy is provided by the balance of energy flux between upstream and downstream vertical surface of this element of length $\Delta \mathrm{l}$.

$$
E_{N}=\rho g C_{g} B T_{w} a_{j} \frac{\partial a_{j}}{\partial x} \Delta l \text {, with : } C_{g}=\frac{\partial \omega}{\partial k}=\frac{g}{2 \omega S_{r}}\left(\tanh \left(k_{j} h\right)+\frac{k_{j} h}{\cosh ^{2}\left(k_{j} h\right)}\right)
$$

$\mathrm{C}_{\mathrm{g}}$ is the group velocity. Finally, the behaviour of wave amplitude in porous media can be obtained by equating both expressions of dissipated energy (eq. 5) and (eq. 6).

$$
\frac{\partial a_{j}}{\partial x}=-C_{d a m p} a_{j}^{2} \text {, with : } C_{d a m p}=-\frac{16}{9 \pi^{2}}(1-\gamma) C_{d, s} \frac{k_{j}}{D} \frac{3+\sinh ^{2}\left(k_{j} h\right)}{\sinh \left(k_{j} h\right) \cosh \left(k_{j} h\right)+k_{j} h}
$$

Assuming $\mathrm{C}_{\mathrm{d}, \mathrm{s}}$ does not depend on the position $\mathrm{x}$, we obtain : $a(x)=a_{0} /\left(a_{0} C_{\text {damp }} x+1\right)$. For the present, $a^{-}(x)=a_{0} /\left(a_{0} C_{\text {damp }} x+1\right)$ and $a^{+}(x)=a(L) /\left(a(L) C_{\text {damp }}(L-x)+1\right)$ with $\mathrm{a}_{0}$ is the wave amplitude at the upstream medium for the incoming wave, downstream for the reflected wave. 
Côtes méditerranéennes menacées :

Risques et défis dans le contexte du changement climatique

\section{Results and discussion}

The presented results focus on wave reflection through the porous media versus a dimensionless parameter $k h$. Experimental data (symbols) and model previsions (lines) are presented in Figure 1 for the three cylinder diameters. The reflection coefficient shows an oscillating behaviour due to interference process between the two successive jumps of medium index at the porous interfaces $x=0$ and $x=L$. The experimental data show a varying pattern of these oscillations for the three cylinder diameters. Indeed, for the smallest cylinder diameter the number of oscillations is more important than for the two other diameters. However, the reflection coefficient fluctuate is the same range $(0.05-0.4)$ for the three diameters at equivalent $k h$. The results of the theoretical model, calibrated on the interference process $\left(\mathrm{C}_{\mathrm{m}}=0.3\right)$ and on the wave damping $\left(\mathrm{C}_{\mathrm{d}, \mathrm{s}}\right)$, give a good representation of reflection coefficients excepted for the smallest diameter. Indeed, the number of oscillations is more important for experimental data than for model previsions but the amplitude range is well predicted.

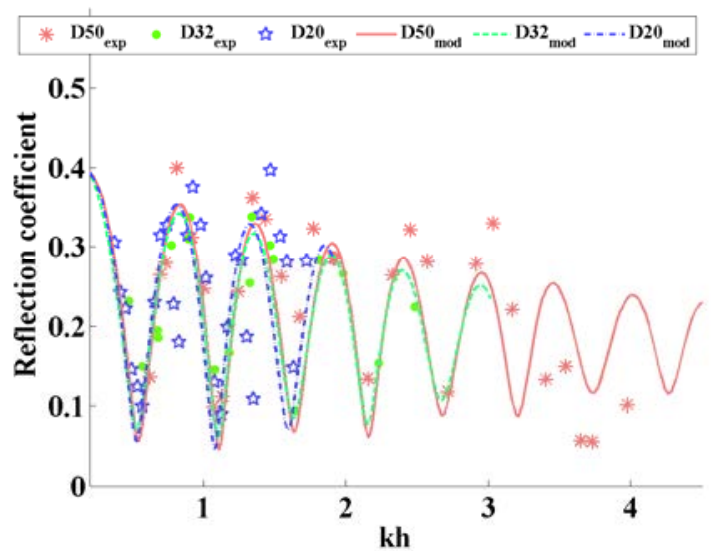

Figure 1. Reflection coefficient versus $k$ for $D=0.050 \mathrm{~m}$ (red), $D=0.032 \mathrm{~m}$ (green) and $D=0.020 \mathrm{~m}$ (blue).

The major differences between the three cylinder diameters can be explained by including our results (Figure 2) in the regime diagram proposed by GU \& WANG (1991) which depicts the Reynolds number $\mathrm{Re}=U D / v$ as a function of the ratio between the Reynolds number and the Keulegan-Carpenter number $K c=U T_{w} / D$. It is first observed that the regimes reached during the present experiments are close to a transition regime. More finely, it appears that for the smallest diameter, the three forces (laminar, turbulent and inertial) are equivalent while for the largest diameter where the laminar effects tend to be negligible for most cases. It is also noted that, for the smallest diameter, water depth is very shallow and the bottom viscosity might become important. 


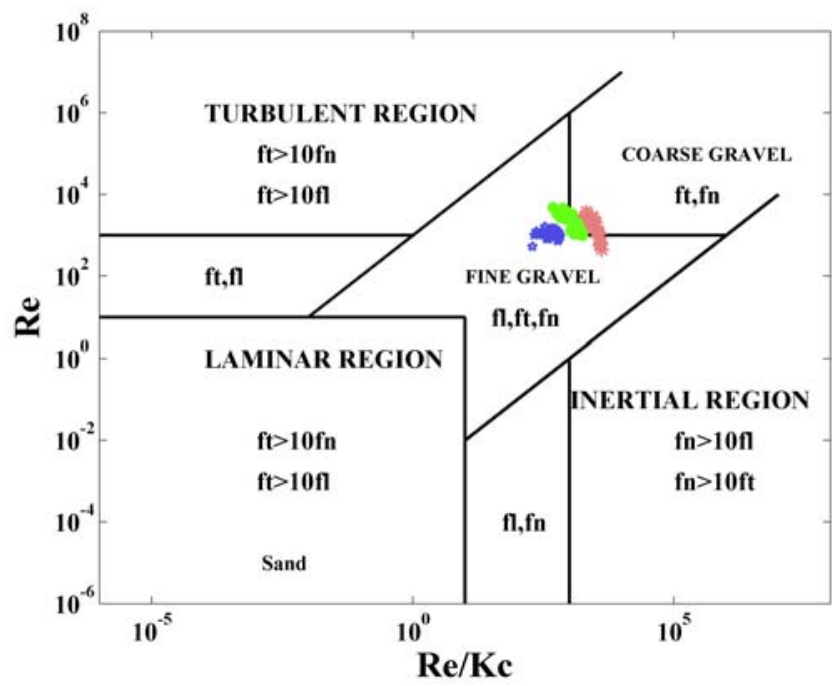

Figure 2. The resistance forces (GU \& WANG, 1991) for $D=0.050 \mathrm{~m}(\mathrm{red}), 0.032 \mathrm{~m}$ (green) and 0.020m (blue).

\section{Conclusion}

This study of scale effects on porous media presents good agreements between experimental data and model prediction. The scaling law study shows the influence of the added mass expressed with the oscillation patterns of reflection coefficient for the three porous media. The energy dissipation due to the propagation through arrays of cylinders is almost similar for the large and intermediate diameters of cylinders in porous media. The limit of results concerns particularly the smallest scale. Indeed, the dissipation through piles is not mostly responsible of wave damping because the water depth is too weak and the bottom effect is not anymore negligible. Further additional studies of scale models are planned to evaluate the irregular wave effects as well as 3D effects.

\section{Acknowledgements:}

The authors would like to thank the "Provence Alpes Côtes-d'Azur" Region and the company "ACRI-IN" for their financial support. A special thank is extended to Bernard MOLIN for his valuable help about the model. This work was supported by ANR grant No ANR-13-ASTR-0007.

\section{References}

ALTOMARE C., GIRONELLA X. (2014). An experimental study on scale effects in wave reflection of low-reflective quay walls with internal rubble mound for regular and random waves. Coastal Engineering, Vol. 90, 51-63. http://dx.doi.org/10.1016/j.coastaleng.2014.04.002 
Côtes méditerranéennes menacées :

Risques et défis dans le contexte du changement climatique

ANDERSEN T.L., BURCHARTH H., GIRONELLA X. (2011). Comparison of new large and small scale overtopping tests for rubble mound breakwaters. Coastal Engineering, Vol. 58(4), 351-373. http://dx.doi.org/10.1016/j.coastaleng.2010.12.004

ARNAUD G., TOUBOUL J., SOUS D., GOUAUD F., REY V. (2014). Ecoulements stationnaires et oscillants à travers des milieux poreux : effets de la surface spécifique. Revue Paralia, Vol. 7, s04.1-s04.12. http://dx.doi.org/10.5150/revue-paralia.2014.s04

DELMONTE R.C. (1972). Scale effects of wave transmission through permeable structures. Coastal Engineering Proceedings, Vol. 1(13), 1867 - 1872.

GU Z., WANG H. (1991). Gravity waves over porous bottoms. Coastal Engineering, Vol. 15, 497-524. http://dx.doi.org/10.1016/0378-3839(91)90025-C

GUYON E., HULIN J.P., PETIT L. (1991). Ecoulements dans les milieux poreux. In Hydrodynamique Physique, Collection Savoirs actuels, interédition/édition du CNRS (pp. 375-384).

O’BRIEN M.P., MORISON J.R. (1952). The Forces Exerted by Waves on Objects.

Transactions, American Geophysical Union, Vol. 33(1), 32-38.

http://dx.doi.org/10.1029/TR033i001p00032

PEREZ-ROMERO D. M., ORTEGA-SANCHEZ M., MOÑINO A., LOSADA M.A. (2009). Characteristic friction coefficient and scale effects in oscillatory porous flow. Coastal Engineering, Vol. 56(9), 931-939. http://dx.doi.org/10.1016/j.coastaleng.2009.05.002

YU X., CHWANG A. (1994). Wave motion through porous structures. Journal of Engineering Mechanics, Vol. 120(5), 989-1008. http://dx.doi.org/10.1061/(ASCE)07339399(1994)120:5(989) 